Sandra Janeth Lagla-Chicaiza; María de Lourdes Llerena-Cepeda

http://dx.doi.org/10.35381/s.v.v4i8.988

\title{
Seguridad del Paciente en Centros de Salud Pública
}

Patient safety in public health centers

\author{
Sandra Janeth Lagla-Chicaiza \\ slagla4714@uta.edu.ec \\ Postgrado de Medicina Familiar y Comunitaria, Facultad de Ciencias de la Salud, \\ Universidad Técnica de Ambato, Ambato \\ Ecuador \\ https://orcid.org/0000-0002-5026-4296 \\ María-de-Lourdes Llerena-Cepeda \\ mdl.Ilerena@uta.edu.ec \\ Postgrado de Medicina Familiar y Comunitaria, Facultad de Ciencias de la Salud, \\ Universidad Técnica de Ambato, Ambato \\ Ecuador \\ https://orcid.org/0000-0002-5721-8006
}

Recepción: 15 de junio 2020

Revisado: 28 de julio 2020

Aprobación: 25 de agosto 2020

Publicación: 03 de septiembre 2020 


\title{
RESUMEN
}

Objetivo: identificar el cumplimiento de la seguridad del paciente en centros de salud del distrito 05D01 Latacunga. Metodología: Descriptiva obsevacional. Se trabajó con 10 centros de salud tipo A, 2 tipo B y 2 tipo C, cuya muestra es de 95 individuos. Resultados: se han identificados los inconvenientes con la seguridad en la atención de salud de pacientes. Conclusión: existen problemas en los sistemas de seguridad en las unidades de salud.

Descriptores: Servicio de admisión en hospital; epidemiología; servicios preventivos de salud. (Fuente DeCS, 2020).

\begin{abstract}
Objective: to identify compliance with patient safety in health centers in the 05D01 Latacunga district. Methodology: Observational descriptive. We worked with 10 type A, 2 type $B$ and 2 type $C$ health centers, whose sample is 95 individuals. Results: drawbacks with safety in patient health care have been identified. Conclusion: there are problems in the security systems in health units.
\end{abstract}

Descriptors: Admitting department, hospital; epidemiology; preventive health services. (Source DeCS, 2020). 


\section{INTRODUCCIÓN}

La seguridad de pacientes permite a los profesionales de salud dar aviso sobre incidentes en las personas, y el análisis de notificaciones facilita el aprendizaje organizacional ${ }^{1}$. Se previenen, si los profesionales siguen los protocolos de seguridad, para reducir la ocurrencia y sus daños, los desperdicios, para que sean efectivos y eficientes ${ }^{2}$.

La codificación de barras, el ingreso de órdenes por sistema y la gestión de recursos son estrategias para prevenir errores en la seguridad del paciente y facilitar los procesos de atención médica. Se conoce la existencia de procedimientos de calidad para la intervención médica; sin embargo, existen errores, se han desarrollado herramientas para la detección de eventos adversos ${ }^{3}$.

Las estadísticas de la Organización Mundial de la Salud (OMS) hablan sobre la seguridad del paciente, por cuanto en países de medios y bajos ingresos, 134 millones de eventos adversos existen al año, son atribuibles a problemas en la atención por errores médicos, diagnósticos equivocados e infecciones adquiridas, provocando 2,6 millones de muertes. El fallecimiento de un paciente por fallas médicas es uno de cada 300 y uno de cada 10 termina con lesiones ${ }^{4}$.

Existe preocupación en la atención de salud en Ecuador y la notificación de los eventos son de carácter obligatorio en la Ley Orgánica de la Salud ${ }^{5}$. Es un problema de salud pública asociado al personal sanitario, las autoridades gubernamentales y los pacientes ${ }^{6}$. Existe el Manual de Seguridad del Paciente que establece procedimientos técnicos, garantizando una adecuada prestación de salud 7 .

Esta investigación tiene como beneficiarios a las personas que asisten a centros de salud del distrito 05D01 y el personal médico. La cultura de seguridad nació en 1988, ha sido adoptado por varias empresas para dar mejoras en su seguridad, cuando hay alto riesgo y necesitan una confiabilidad extrema. Se reduce la ocurrencia de eventos adversos, cuyo compromiso es la seguridad en todos sus niveles. Las unidades de salud se enfocan en la "seguridad en el paciente", con recursos y conductas del personal 8. 
En el estudio de Rocco y Garrido ${ }^{9}$, el mejoramiento de la cultura de seguridad es fundamental previniendo la reducción de fallas, y habla sobre la calidad de atención médica. La interconexión entre personas, los sistemas y el aprendizaje, ayudan a la seguridad del paciente y la gestión de incidentes.

Los componentes de esta cultura son de aprendizaje: estudia los errores de seguridad identificados; de justicia: se ajustan a la confianza, la equidad y la responsabilidad; y de informes: facilita la notificación de fallas, y ayuda a corregirlos ${ }^{10}$. Exige sistemas que ofrezcan confiabilidad en las intervenciones. Se analizan su complejidad, si es mayor, aumenta la propensión al caos. Estos requieren de diseño de materiales, medio ambiente, procedimientos, capacitación, así como la naturaleza de cultura ${ }^{11 .}$

En este sentido se tiene por objetivo de investigación identificar el cumplimiento de la seguridad de los pacientes en los centros de salud públicos del distrito 05D01 Latacunga.

\section{MATERIAL Y MÉTODOS}

\section{Diseño}

Diseño no experimental descriptivo observacional analítico.

\section{Población de investigación}

Conformada por 95 pacientes de centros de salud públicos del distrito 05D01 Latacunga. Se trabajó con 10 centros de salud tipo A, 2 tipo B y 2 tipo C.

\section{Ámbito espacial de la investigación}

Se desarrolló en centros de salud públicos del distrito 05D01 en San Vicente Mártir de Latacunga, Provincia de Cotopaxi - Ecuador. 


\section{Instrumentos de recolección de datos}

Se aplicó encuesta e instrumento tipo cuestionario en escala de Likert de 13 ítems de varias alternativas de respuestas, siendo validado por juicio de expertos y cálculo de coeficiente Alfa de Cronbach, teniendo un resultado de 0,87 .

\section{Análisis de datos}

Los datos obtenidos fueron procesados en SPSS y Excel Microsoft Office, gestionando campos para la variable en estudio.

\section{RESULTADOS}

El personal de salud encuestado tiene una edad comprendida entre 23 a 33 años (73\%), seguidas de 34 a 54 años en adelante. El personal femenino predomina en estos centros de salud $(78 \%)$, mientras que el $22 \%$ corresponde al género masculino. Los puestos profesionales corresponden a médicos (34\%), enfermeras (21\%), licenciados (21\%), odontólogos (16,84\%) y obstetras $(7,37 \%)$.

El 39\% del personal de salud están de acuerdo que existen procesos de promoción de seguridad del paciente, sin embargo, estos necesitan procedimientos, así como el compromiso del personal. Un 35\% están totalmente de acuerdo y un $23 \%$ no están de acuerdo ni en desacuerdo. 
Revista Arbitrada Interdisciplinaria de Ciencias de la Salud. SALUD Y VIDA

Volumen 4. Número 8. Año 4. Julio-Diciembre 2020

Hecho el depósito de Ley: FA2016000010

ISSN: 2610-8038

FUNDACIÓN KOINONIA (F.K).

Santa Ana de Coro, Venezuela.

Sandra Janeth Lagla-Chicaiza; María de Lourdes Llerena-Cepeda

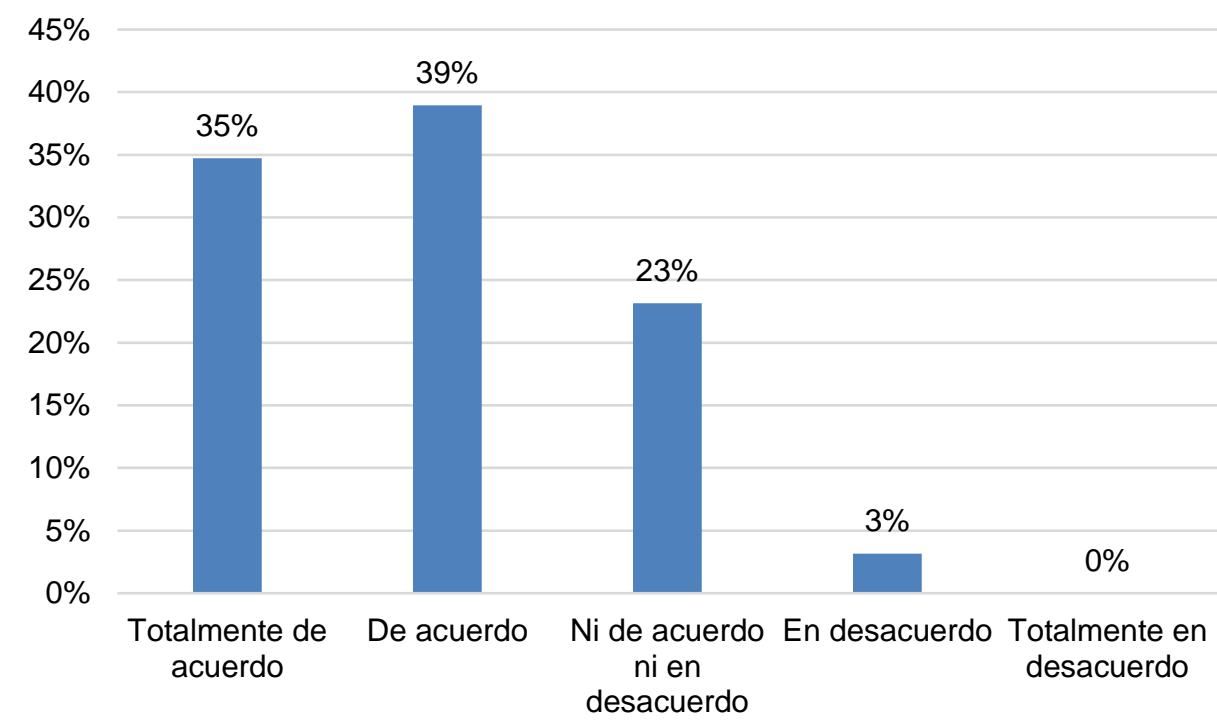

Figura 1. Promoción de seguridad del paciente en su área de trabajo.

El personal de salud calificó a los sistemas de seguridad del paciente, el $65 \%$ los considera regulares y el $5 \%$ los catalogan como excelentes.

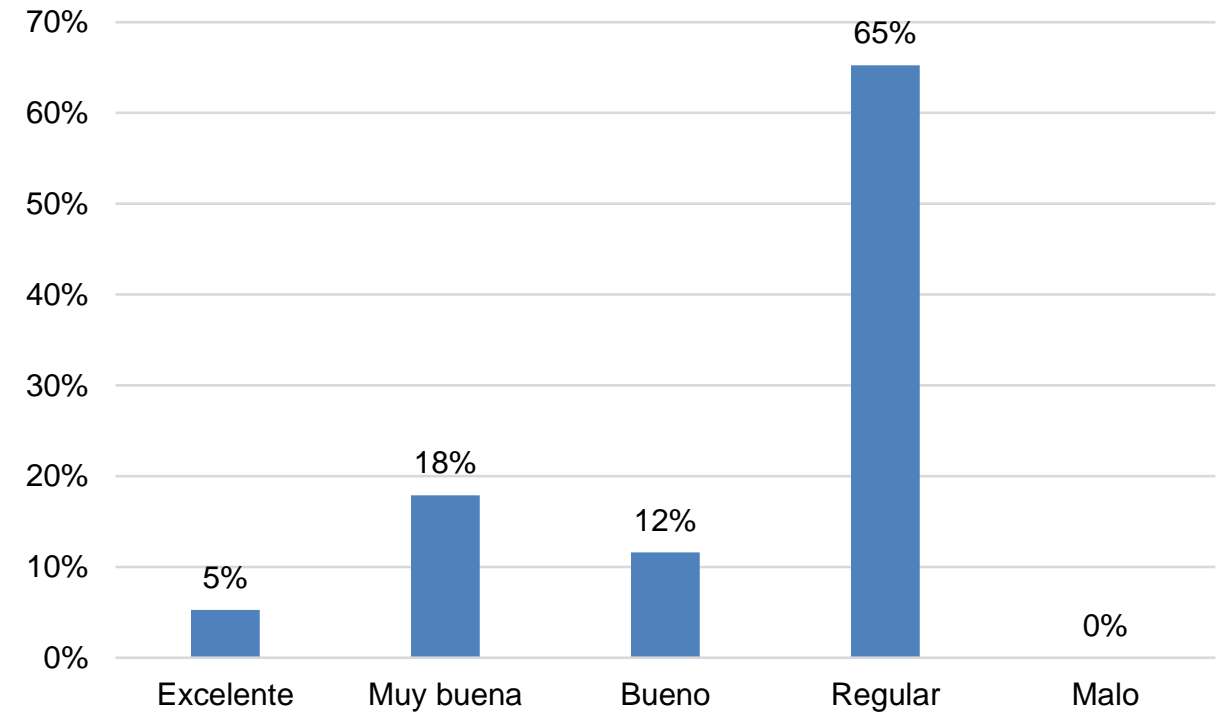

Figura 2. Calificación a los procedimientos y sistemas aplicados. 
Para mejorar la calidad de la seguridad de los pacientes, las acciones más eficientes son aumentar el nivel de conocimiento (66\%), y disponer de un profesional que analice esta problemática (34\%).

Los profesionales están de acuerdo en que hay problemas en las áreas de trabajo relacionados con la seguridad y la atención de la salud de los pacientes (39\%), mientras que el $32 \%$ piensan que no hay inconvenientes y un $24 \%$ desconocen si hay novedades referentes a estos procesos.

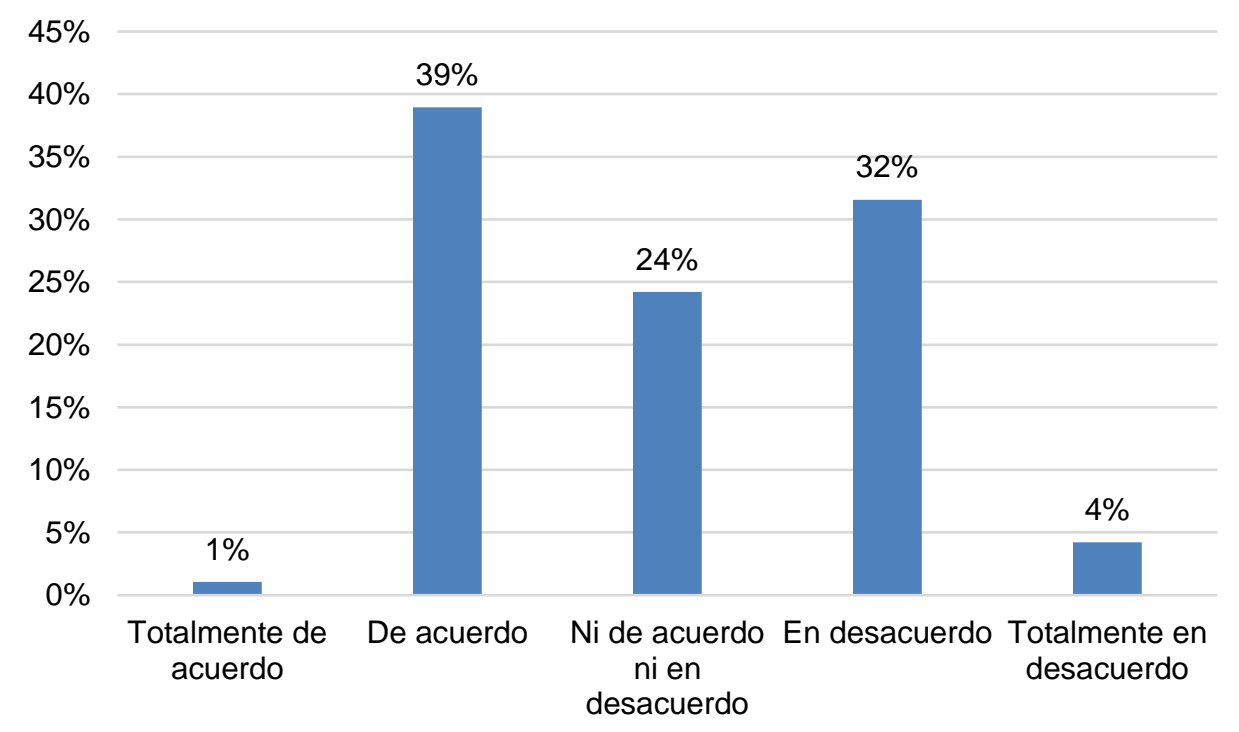

Figura 3. Problemas en el área de trabajo

La frecuencia de actividades para mejorar la seguridad de los pacientes se evidencia en un $53 \%$, ya que rara vez son realizadas. El $31 \%$ consideran que a veces se las desarrollan, el $14 \%$ expresan que siempre se han realizado, mientras que el $2 \%$ nunca las han visto. 
Revista Arbitrada Interdisciplinaria de Ciencias de la Salud. SALUD Y VIDA

Volumen 4. Número 8. Año 4. Julio - Diciembre 2020

Hecho el depósito de Ley: FA2016000010

ISSN: $2610-8038$

FUNDACIÓN KOINONIA (F.K)

Santa Ana de Coro, Venezuela.

Sandra Janeth Lagla-Chicaiza; María de Lourdes Llerena-Cepeda

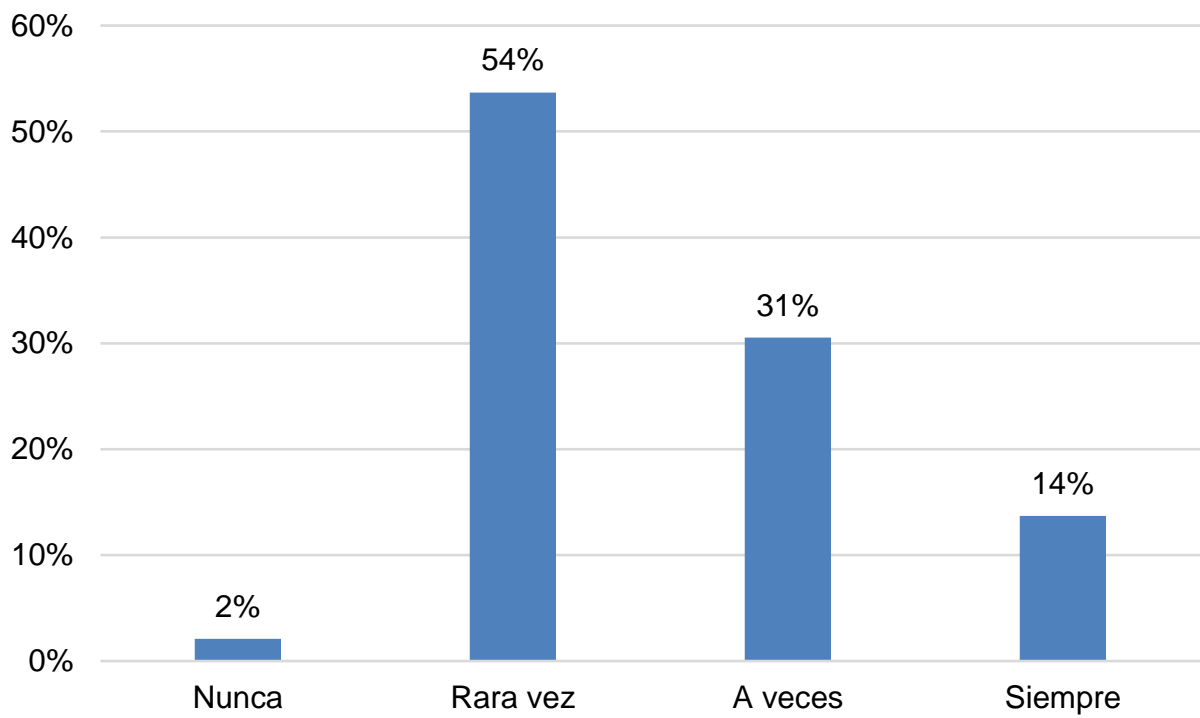

Figura 4. Seguridad de los pacientes en el centro de salud.

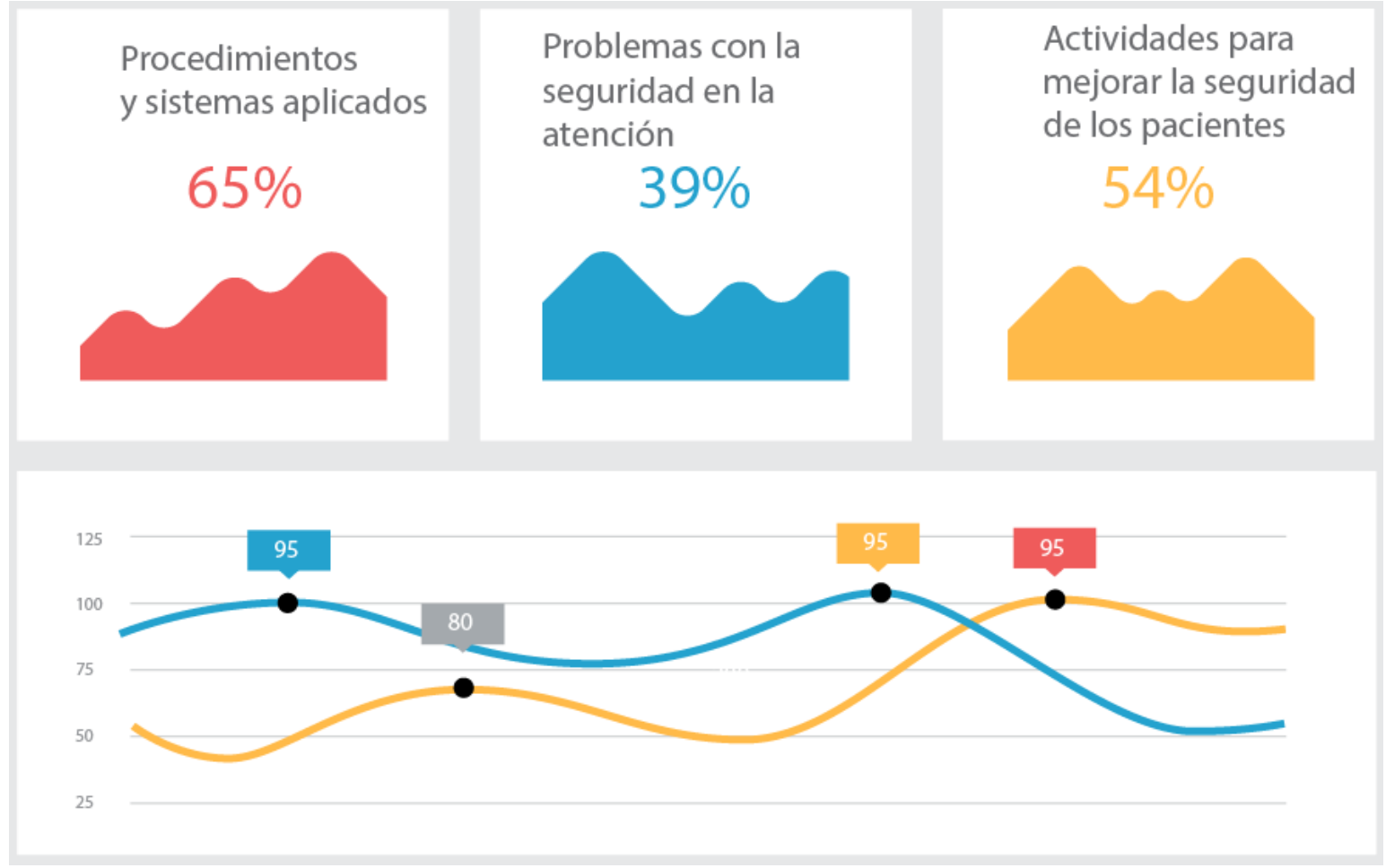

Figura 5. Representación gráfica general de los resultados. 


\section{DISCUSIÓN}

El personal de salud calificó a los sistemas de seguridad y atención de los pacientes, como regulares (65\%) y el $5 \%$ son de excelencia. Se necesita fortalecer los procesos, existen nuevas redes de atención primaria que ayudan a la promoción y mejora continua. Pese a la existencia de procedimientos, no son acatados por los funcionarios, debido al desconocimiento, el inadecuado interés y la falta de capacitación.

El estudio de ${ }^{12}$ identifica metodologías para incidentes en la atención de salud y los factores que ayudan a su solución. Los incidentes están asociados a la medicación y métodos de diagnóstico. El aspecto más significativo es la falta de comunicación.

El estudio de ${ }^{13}$ considera realizar cambios en los sistemas de salud, como mejorar la supervisión, ajuste en la carga laboral y actividades que prevengan la fatiga para aumentar el desempeño de sus funcionarios.

La afectación de la seguridad de los pacientes en la atención y la notificación de eventos adversos, se debe por el desconocimiento de reportes de eventos, que son tomados con poca seriedad por los profesionales; trabajo excesivo; poca disponibilidad de tiempo ${ }^{14}$. El estudio realizado por ${ }^{15}$ identificó las debilidades en la seguridad de pacientes, 41 enfermeras de instituciones de salud en Bucaramanga, Ilenaron un cuestionario. Se encontraron problemas económicos, años de trabajo, edad, horarios laborales. Están conscientes del compromiso y de la existencia de procedimientos en los centros médicos. En los centros de salud públicos del distrito 05D01 Latacunga existen problemas en el cumplimiento de la seguridad del paciente, asociados directamente con la calidad de atención. Los profesionales de salud no realizan las notificaciones de los eventos adversos, por el desconocimiento del sistema y procedimientos de salud (calificados como regulares), y la poca disponibilidad de tiempo por sobrecarga de trabajo. El grado de seguridad de los pacientes que asistente a estas casas de salud es bueno, sin embargo, los centros de salud presentan problemas. 
Para incentivar la cultura de seguridad, es necesario incluir informes que evidencien los errores en el proceso de atención para su análisis, aportando en la toma de decisiones sobre políticas de salud y desarrollar programas de información dirigidos a los usuarios.

\section{CONFLICTO DE INTERÉS}

La investigación apunta a mejorar la seguridad y la atención de pacientes, fue supervisada por las autoridades de las unidades de salud para evitar recibir incentivos o coimas que alteren la información recolectada.

\section{FINANCIAMIENTO}

La investigación se realizó con el apoyo de los centros de salud del distrito 05D01.

\section{AGRADECIMIENTO}

Al Centro de posgrado de la Universidad Técnica de Ambato. Especialización en Medicina Familiar y Comunitaria, segunda cohorte; por el apoyo en el desarrollo de la investigación.

\section{REFERENCIAS}

1. World Health Organization. Más que palabras. Marco Conceptual de la Clasificación Internacional para la Seguridad del Paciente. [More than words. Conceptual Framework of the International Classification for Patient Safety]. Informe Técnico Definitivo Enero de 2009 [Internet]. OMS. 2009. 1-160 p. Recuperado de https://n9.cl/mfb3

2. Jácome J, González O. Caracterización de eventos adversos por errores de dispensación reportados en el programa distrital de fármaco vigilancia de Bogotá D.C. 2012-2016. [Characterization of adverse events due to dispensing errors reported in the district drug surveillance program of Bogotá D.C. 2012-2016]. Univ Ciencias Apl y Ambient [Internet]. 2017;1-95. Recuperado de https://n9.cl/2vu43 
3. Agencia Nacional de Regulación Control y Vigilancia Sanitaria. Plan Anual Comprometido 2019 [Internet]. 2020. 1-46 p. Recuperado de https://n9.cl/q411q

4. Prado M, Rizo A, Díaz P, Hoyos A. Seguridad del paciente un compromiso institucional para una atención más segura. Univ Católica Manizales [Internet]. 2015;1-258. Recuperado de https://n9.cl/ie5i

5. Zárate R. Validación del instrumento "Factores asociados a la notificación de eventos adversos por el personal de salud." Rev Enferm Inst Mex Seguro Soc [Internet]. 2017;25 (3):227-32.

6. Murff H, Patel V, Hripcsak G, Bates D. Detecting adverse events for patient safety research: a review of current methodologies. Natl Libr Med [Internet]. 2003;36 (12)(1):31-43.

7. Hospital Vicente Corral Moscoso. Norma técnica de seguridad del paciente [Internet]. 2015. p. 1-85. Recuperado de https://n9.cl/219dr

8. Vítolo F. Cultura de Seguridad. Bibl Virtual NOBLE [Internet]. 2016; 1-13. Recuperado de https://n9.cl/s8hl

9. Rocco C, Garrido A. Seguridad del paciente y cultura de seguridad. Rev Médica Clínica Las Condes [Internet]. 2017;28(5):785-95. http://dx.doi.org/10.1016/j.rmclc.2017.08.006

10. Ramírez M, González A. Cultura de seguridad y eventos adversos en una clínica de primer nivel. Enfermería Univ [Internet]. 2017;14(2):111-7. http://dx.doi.org/10.1016/j.reu.2017.02.006

11. Astier M, Torijano M, Olivera G. Setting priorities for patient safety in Primary Care. Aten Primaria [Internet]. 2016; 48(1):3-7.

12. Marchon S, Mendes W. Patient safety in primary health care: a systematic review. Cad Saude Publica. 2014;30(9):1815-35.

13. Gaviria C. Percepción del clima de seguridad del paciente por el personal de salud en la Institución de primer nivel de complejidad ESE Centro de Salud de Tota. Univ St Tomas [Internet]. 2017; (1):1-31. Recuperado de http://hdl.handle.net/11634/18340 
Revista Arbitrada Interdisciplinaria de Ciencias de la Salud. SALUD Y VIDA

Volumen 4. Número 8. Año 4. Julio - Diciembre 2020

Hecho el depósito de Ley: FA2016000010

ISSN: 2610-8038

FUNDACIÓN KOINONIA (F.K).

Santa Ana de Coro, Venezuela.

Sandra Janeth Lagla-Chicaiza; María de Lourdes Llerena-Cepeda

14. Lawati M, Dennis S, Short S, Abdulhadi N. Patient safety and safety culture in primary health care: A systematic review. BMC Fam Pract [Internet]. 2018;19(104):1-12.

15. Camacho $D$, Jaimes $N$. Cultura de seguridad del paciente en enfermeros de atención primaria. Rev Cubana Enferm [Internet]. 2018;34(1):1-8.

C2020 por los autores. Este artículo es de acceso abierto y distribuido según los términos y condiciones de la licencia Creative Commons Atribución-NoComercial-Compartirlgual 4.0 Internacional (CC BY-NC-SA 4.0)

(https://creativecommons.org/licenses/by-nc-sa/4.0/). 